\title{
Fourier Spectral Method for Solving Fractional-order System
}

\author{
Kolade M. Owolabi ${ }^{1, *}$, Ayodeji A. Adejola ${ }^{2}$ \\ ${ }^{1}$ Department of Mathematical Sciences, Federal University of Technology, PMB 704, Akure, Ondo State, Nigeria \\ ${ }^{2}$ Department of Computer Engineering, Federal University of Technology, PMB 704, Akure, Ondo State, Nigeria
}

Copyright $\odot 2017$ by authors, all rights reserved. Authors agree that this article remains permanently open access under the terms of the Creative Commons Attribution License 4.0 International License

\begin{abstract}
In this paper, we have studied a new fractional reaction-diffusion two-species system as an extension to the Rosenzweig-MacArthur reaction-diffusion di-trophic food chain system which models the spatial interactions between a prey and predator. To guarantee good working guidelines when numerically simulating the model, we first show that the system is locally asymptotically stable, as it provides good conditions and correct choice of ecological parameters to enhance a biologically meaningful result. We propose a fast and accurate method for numerical solutions of space fractional reactiondiffusion equations. The technique is based on Fourier spectral method in space and exponential integrator scheme in time. The complexity of fractional derivative index in fractional reaction diffusion model is numerically formulated and graphically displayed in one-, two- and three-dimensions.
\end{abstract}

Keywords Fourier Spectral Method, Exponential Integrator, Reaction-diffusion System, Oscillations, Rosenzweig-macarthur Model, Stability Analysis

2010 Mathematics Subject Classification: 34A34, 35A05, 35K57, 65L05, 65M06, 93C10

\section{Introduction}

Historically, the earlier mathematical approach for two-variable system was reported to model spatial interactions of predator-prey dynamics of the Lotka-Volterra type. It had since become a good testing ground for describing population dynamics. Years later, more realistic and interesting two-species models were proposed. For instance, May [16] proposed in a model

$$
\left.\begin{array}{l}
\frac{d u}{d t}=\tau u\left(1-\frac{u}{\kappa}\right)-\sigma u v, \\
\frac{d v}{d t}=-\sigma u+\tau u v,
\end{array}\right\}
$$

that the prey population grows logistically. The linear stability analysis result of (1) [5] indicates that the coexistence equilibrium, dependent of the carrying capacity $\kappa$ of the prey population density is always a stable point. Owing to the assumption that the predator-prey model underlies a saturation, we have a model attributed to Rosenzweig-MacArthur di-trophic food chain system $[6,29]$

$$
\left.\begin{array}{l}
\frac{d u}{d t}=\tau u\left(1-\frac{u}{\kappa}\right)-\sigma_{2} \frac{u}{\rho_{1}+u} v, \\
\frac{d v}{d t}=\gamma_{2} \sigma_{2} \frac{u}{\rho_{1}+u} v-\delta_{2} v,
\end{array}\right\}
$$

where $u$ and $v$ denote the population of prey and predator respectively for $t>0$, measure as a number of individuals, biomass or density, $\tau$ represents the prey biotic potential or intrinsic growth rate, $\kappa$ is the environmental carrying capacity of the prey, 
$\sigma_{2}$ defines the predator maximum predation rate, $\gamma_{2}$ is the predator efficiency and $\rho$, is known to be half saturation amount (the rate of prey required to get one-half of $\sigma_{2}$ ). The death rate of predator is denoted by $\delta_{2}$.

The reaction-diffusion model (2) has been shown in [38] to have a unique and globally stable attractor. A lot of twospecies models with various functional responses or Holling type-II, reaction-diffusions, and delays have been reported by many researchers (see, for instance[2, 30, 31, 34] and references therein). In recent times, researchers have shown a great deal of interest in the study of fractional reaction-diffusion systems [1, 3, 9, 10, 12, 15, 24, 26, 35, 36, 39, 40, 41], and some classical textbooks $[13,27,28,42]$.

In this paper, we begin our study with space fractional diffusion equation

$$
\frac{\partial}{\partial t} \mathcal{U}(\wp, t)=\delta \frac{\partial^{\beta}}{\partial \wp^{\beta}} \mathcal{U}(\wp, t), \quad \infty<\wp<\infty, \quad 0 \leq t<\infty, \quad 0 \leq \beta
$$

where $\mathcal{U}(\wp, t)$ is the species population density distribution at positions $\wp=\wp(x, y, z)$ and time $t$, it could also be regarded as the concentration of chemical species. The space fractional operator $\delta \frac{\partial^{\beta}}{\partial \wp^{\beta}}(\wp, t), 0<\beta<2$ is given on the basis of the Fourier transformation, with variable $\delta>0$ as the diffusion coefficient. Here, we define the Fourier transformation and its corresponding inverse as

$$
\begin{aligned}
\hat{F}(k)=\mathcal{F}\{F(\wp)\} & =\int_{-\infty}^{\infty} F(\wp) e^{i k \wp} d \wp, \\
F(\wp)=\mathcal{F}^{-1}\{\hat{F}(k)\} & =\frac{1}{2 \pi} \int_{-\infty}^{\infty} \hat{F}(k) e^{-i k \wp} d k .
\end{aligned}
$$

Next, we apply the space fractional operator on the species population density $\mathcal{U}(\wp, t)$ defined on $-\infty<\wp<\infty$, $0 \leq t<$ $\infty, \quad 0 \leq \beta$ to yield

$$
\begin{aligned}
\frac{\partial^{\beta}}{\partial \wp^{\beta}} \mathcal{U}(\wp, t) & =\mathcal{F}^{-1}\left\{(-i k)^{\beta} \mathcal{F}\{\mathcal{U}(\wp, t)\}\right\}, \\
\frac{\partial^{\beta}}{\partial(-\wp)^{\beta}} \mathcal{U}(\wp, t) & =\mathcal{F}^{-1}\left\{(i k)^{\beta} \mathcal{F}\{\mathcal{U}(\wp, t)\}\right\} .
\end{aligned}
$$

Prior to definition in (5), Fourier transform of (5) results to an ordinary differential equation (ODE) of the form

$$
\frac{\partial}{\partial t} \hat{\mathcal{U}}(\wp, t)=\delta(-i k)^{\beta} \hat{\mathcal{U}}(k, t)
$$

subject to initial function

The solution of (6) is sought as

$$
\mathcal{U}(\wp, 0)=f(\wp), \quad \hat{\mathcal{U}}(k, 0)=\mathcal{F}\{\mathcal{U}(\wp, 0)=\mathcal{F}\{f(\wp)\}=1 .
$$

$$
\hat{\mathcal{U}}(k, t)=e^{\delta t(-i k)^{\beta}} \hat{\mathcal{U}}(k, 0)=e^{\delta t(-i k)^{\beta}}
$$

where

$$
\begin{aligned}
(-i k)^{\beta} & =\left[|k| e^{i \arg (-i k)}\right]^{\beta}=|k|^{\beta} e^{i \beta\left(-\operatorname{sign}(k) \frac{\pi}{2}+2 n \pi\right)} \\
& =|k|^{\beta} \cos \left(\frac{\beta \pi}{2}\right)\left[1-i \operatorname{sign}(k) \tan \left(\frac{\beta \pi}{2}\right)\right],
\end{aligned}
$$

which results to

$$
\hat{\mathcal{U}}(k, t)=e^{\delta t|k|^{\beta}} \cos \left(\frac{\beta \pi}{2}\right)\left[1-i \operatorname{sign}(k) \tan \left(\frac{\beta \pi}{2}\right)\right],
$$

with corresponding inverse Fourier transform

$$
\mathcal{U}(k, t)=\mathcal{F}^{-1}\left\{e^{\delta t|k|^{\beta}} \cos \left(\frac{\beta \pi}{2}\right)\left[1-i \operatorname{sign}(k) \tan \left(\frac{\beta \pi}{2}\right)\right]\right\} .
$$

Readers are referred to classical books [13, 27, 28, 42] for details of the preliminary theories and definitions of fractional derivatives.

The aims of the present paper are in folds; In Section 2, we give an extension to the commonly two-variable RosenzweigMacarthur reaction-diffusion system and examine the linear stability analysis of the new model. In Section 3, we formulate a good versatile numerical techniques based on Fourier spectral method in space and exponential time differencing method for simulating the fractional reaction-diffusion problem of Rosenzweig-MacArthur type with delay in one and two dimensions. Numerical experiments and concluding remarks are given in Sections 4 and 5 respectively. 


\section{Mathematical analysis of the space fractional Rosenzweig-MacArthur model with delay}

In this work, we give an extension to model (2) in the form of fractional reaction-diffusion system

$$
\left.\begin{array}{l}
\partial_{t} u-D_{u} \Delta^{\beta}=f(u, v)=u\left(1-\frac{u}{\kappa}\right)-\sigma \frac{u}{1+u} v, 0 \leq \beta \leq n, n \in \mathbf{R} \\
\partial_{t} v-D_{v} \Delta^{\beta}=g(u, v)=\sigma \frac{u(t-\tau)}{1+u(t-\tau)} v(t-\tau) \exp (\gamma \tau)-\delta v,
\end{array}\right\}
$$

subject to the initial condition

$$
u(\wp, 0)=u_{0}(\wp), \quad v(\wp, 0)=v_{0}(\wp), \quad \wp \in \Omega
$$

and the zero-flux boundary condition

$$
\frac{\partial u}{\partial \nu}=\frac{\partial v}{\partial \nu}=0, \quad \wp \in \partial \Omega, \quad t>0
$$

where $\nu$ is the outward normal to to the boundary $\partial \Omega, u(\wp, t) \in \mathbf{R}$ and $v(\wp, t) \in \mathbf{R}$ are the respective population densities of prey $u$ and predators $v$ at time $t$ and position $\mathbf{x}$. Parameters $\sigma, \tau, \gamma$ and $\delta$ are strictly positive, $D_{u}>0$ and $D_{v}>0$ are the prey-predator diffusion coefficients. The parameter $\beta>0$ is the fractional power that distinguishes between the classical and fractional reaction-diffusion systems. If $\beta=1$, equation (7) reduces to the classical reaction-diffusion system. If $0<\beta<1$, we have a sub-diffusive case and if $\beta>1$, a situation that corresponds to super-diffusive system is attained.

In order to give a good working versatile guidelines when numerically simulating the full fractional reaction-diffusion system, it is important to provide details of the local dynamics of such system. local analysis provides appropriate conditions and good choice of parameters for the solutions to have a biologically meaningful equilibria. We shall focus on the dynamics of interest in the region $u \geq 0, v \geq 0$ that is biologically meaningful. The nullclines are the solution curves that correspond to the equations $f(u, v)=0, g(u, v)=0$.

Linear stability analysis reveals that system (7) is trivially satisfied if $\tau=0$, a point that corresponds to total extinction of both species, so the trivial equilibrium point $(0,0)$ is a saddle. We are not interested in the trivial result because it is of no interest biologically. Hence, we are primarily concerned with an equilibrium state which is either stable or unstable corresponding to the coexistence of both species. The point is given by $E_{s}=(\hat{u}, \hat{v})$ with

$$
\hat{u}=\frac{\delta}{\sigma-\delta}, \quad \hat{v}=\left(1-\frac{\delta}{\kappa(\sigma-\delta)}\right) \frac{1}{\sigma-\delta}, \text { for } \sigma>\delta, \text { and } \kappa>H_{a}=\frac{\delta}{\sigma-\delta} .
$$

The equilibrium point $E_{s}$ is locally asymptotically stable if $\kappa<(\sigma+\delta) /(\sigma-\delta)$, the point becomes unstable when a Hopf bifurcation occurs at $H_{b}=(\sigma+\delta) /(\sigma-\delta)$. Consider the case $\tau>0$, for $\sigma>\delta \exp (\gamma \tau)$ and $\kappa<\delta /(\sigma \exp (-\gamma \tau)-\delta)$, a point corresponding to the existence of only prey, that is $E_{1}=(\kappa, 0)$, which is stable for all values of $\tau \geq 0$. So also, for $\sigma>\delta \exp (\gamma \tau)$ and $\kappa>\delta /(\sigma \exp (-\gamma \tau)-\delta)$, there is another semi-trivial state $E_{2}=(0, \kappa)$ which corresponds to the predators existence. There is also one nontrivial steady state

$$
E_{s}=(\hat{u}, \hat{v})=\left(\frac{\delta}{\sigma e^{-\gamma \tau}-\delta}, \frac{1}{\sigma}\left(1-\frac{\hat{u}}{\kappa}\right)(1+\hat{u})\right)
$$

where the two species coexist, and whose stability depends on parameter $\tau$. We find the parameter set in which stability can occur as:

$$
\psi=\frac{2 \delta}{\kappa\left(\sigma e^{-\gamma \tau}-\delta\right)}-e^{\gamma \tau}\left(\frac{\delta}{\sigma}+\frac{\delta}{\kappa \sigma}\right), \phi=\delta e^{\gamma \tau}\left(1-e^{\gamma \tau}\left(\frac{\delta}{\sigma}+\frac{\delta}{\kappa \sigma}\right)\right), \varphi=\delta e^{\gamma \tau} .
$$

We know that the stability of point $E_{s}$ depends on the value of the delay $\tau \geq 0$. Stability properties of $E_{s}$ are found by the real part of the characteristic roots, such that

$$
\varphi-2 \delta e^{\gamma \tau} \psi>0 \Longrightarrow 1+e^{\gamma \tau}\left(\frac{\delta}{\sigma}+\frac{\delta}{\kappa \sigma}\right)-\frac{4 \delta}{\kappa\left(\sigma e^{-\gamma \tau}-\delta\right.}>0
$$

and

By simplifying (8), we obtain

$$
\lambda^{2}+\frac{3 \sigma}{\delta(1+\kappa)} \lambda-\frac{\kappa \sigma^{2}}{\delta^{2}(1+\kappa)} \lambda<0
$$

$$
\lambda_{1,2}=-\frac{9 \sigma^{2}}{2 \delta(1+\kappa)} \pm \sqrt{\frac{3 \sigma}{4 \delta(1+\kappa)^{2}}+\frac{\kappa \sigma^{2}}{\delta^{2}(1+\kappa)}}
$$


It should be noted that both $\tau$ and $\kappa$ can be chosen as bifurcation parameters for system (7). for instance, for $\tau=0$ and $\kappa \in\left(0, H_{a}\right)$ the point $E_{2}$ is stable, and the coexistence point $E_{s}$ is locally asymptotically stable for $\kappa \in\left(H_{a}, H_{b}\right)$. Whenever $\kappa \gg H_{a}$, a limit cycle occurs. It is noticeable that the destabilization of the equilibria is shift forward due to inclusion of a delay. That is, if $\tau>0$ and $\kappa \in\left(0, E_{\hat{u}}(\tau)\right)$, with $E_{\hat{u}}(\tau) \leq E_{\hat{u}}(0)=H_{a}$, then the point $E_{2}$ is stable, but if $\kappa \in\left(E_{\hat{u}}(\tau), E_{\hat{s}}(\tau)\right)$, with $E_{\hat{s}}(\tau) \leq E_{\hat{s}}(0)=H_{b}$, then the coexistence equilibrium state $E_{s}$ is locally asymptotically stable. it should also be noted that an increase in the delay may give rise to damped oscillations whenever $\kappa>E_{\hat{s}}(\tau)$, as depicted in Figure 1.
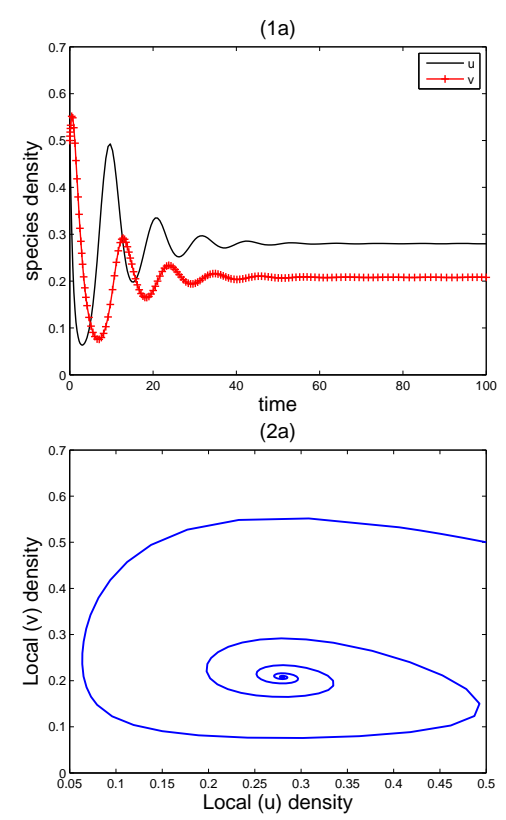

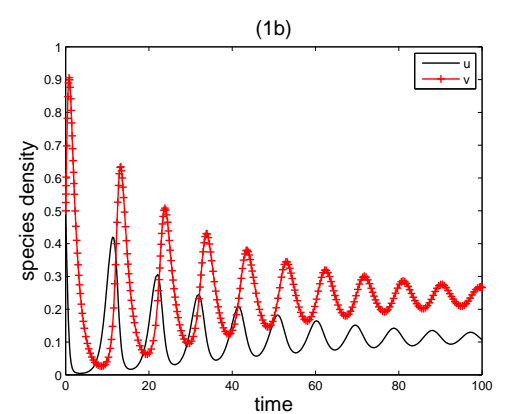

(2b)

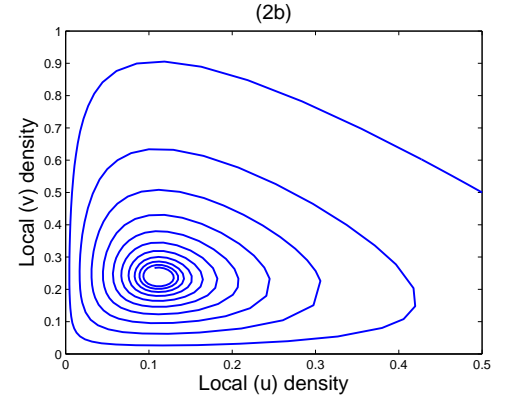

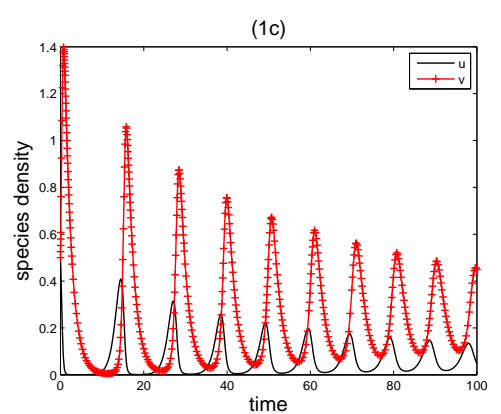

(2c)

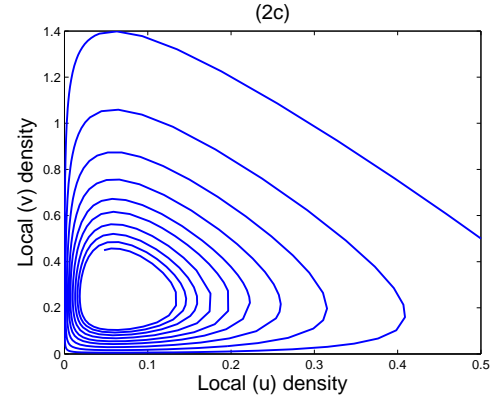

Figure 1. Effect of the delay on the solution of dynamical model (7) at different instants of $\tau$. The ecological parameters and initial population data are chosen as: $\kappa=0.8, \sigma=4, \gamma=0.4$ and $\delta=0.6$ for $\left(u_{0}, v_{0}\right)=(0.5,0.5)$ at $t=100$.

\section{Numerical technique for the fractional reaction-diffusion system}

The fractional reaction-diffusion Rosenzweig-MacArthur system (7) supports the use of two classical methods, since the problem can be divided into the linear and nonlinear parts [33, 37]. As a result, we formulate a Fourier spectral method for the spatial discretization and employ higher order exponential time differencing scheme to advance the resulting system of ordinary differential equations in time.

\subsection{Formulation of adaptive methods in space and time}

In recent years, credit has been given to spectral methods over the conventional finite differences [7, 8, 20], due to its capability of removing the stiffness issue associated with the of a reaction-diffusion problems. From the known theory of integrating factor technique, we shall formulate the spectral method in two spatial dimensions. In the spirits of [20, 25], we apply the integrating factor technique to the Fourier transform systems (7), to obtain

$$
\begin{aligned}
& U_{t}\left(\chi_{x}, \chi_{y}, t\right)=\left[-\left(\chi_{x}^{\beta}+\chi_{y}^{\beta}\right) U\left(\chi_{x}, \chi_{y}, t\right)\right]^{\beta}+\mathcal{F}[f(u(x, y, t), v(x, y, t))] \\
& V_{t}\left(\chi_{x}, \chi_{y}, t\right)=\left[-\delta_{1}\left(\chi_{x}^{\beta}+\chi_{y}^{\beta}\right) V\left(\chi_{x}, \chi_{y}, t\right)\right]^{\beta}+\mathcal{F}[g(u(x, y, t), v(x, y, t))]
\end{aligned}
$$

where $U$ and $V$ are the double Fourier transforms for the species densities $u(x, y, t), v(x, y, t)$. In other words,

$$
\begin{aligned}
\mathcal{F}[u(x, y, t)] & =U\left(\chi_{x}, \chi_{y}, t\right)=\int_{-\infty}^{\infty} \int_{-\infty}^{\infty} u(x, y, t) e^{-i\left(\chi_{x} x+\chi_{y} y\right)} d x d y \\
\mathcal{F}[v(x, y, t)] & =V\left(\chi_{x}, \chi_{y}, t\right)=\int_{-\infty}^{\infty} \int_{-\infty}^{\infty} v(x, y, t) e^{-i\left(\chi_{x} x+\chi_{y} y\right)} d x d y .
\end{aligned}
$$

To explicitly circumvent the issue of stiffness in the second partial derivatives, we let $\Omega^{\beta}=\left(\chi_{x}^{\beta}+\chi_{y}^{\beta}\right)$, and set

$$
U=e^{-\Omega^{\beta} t} \bar{U}, V=e^{-\delta_{1} \Omega^{\beta} t} \bar{V}
$$


so that

$$
\begin{aligned}
\partial_{t} \bar{U} & =e^{\Omega^{\beta} t} \mathcal{F}[f(u, v)], \\
\partial_{t} \bar{V} & =e^{\delta_{1} \Omega^{\beta} t} \mathcal{F}[g(u, v)] .
\end{aligned}
$$

Next, we need to discretize the square domain by considering the equispaced points $N_{x}$ and $N_{y}$ in the spatial directions of $x$ and $y$. We use the discrete fast Fourier transform (DFFT) [33] to transform (9) to a system of ordinary differential equations (ODEs)

$$
\begin{aligned}
\partial_{t} \bar{U}_{i, j} & =e^{\Omega_{i, j}^{\beta} t} \mathcal{F}\left[f\left(u_{i, j}, v_{i, j}\right)\right], \\
\partial_{t} \bar{V}_{i, j} & =e^{\delta_{1} \Omega_{i, j}^{\beta} t} \mathcal{F}\left[g\left(u_{i, j}, v_{i, j}\right)\right],
\end{aligned}
$$

where $u_{i, j}=u\left(x_{i}, y_{j}\right), v_{i, j}=v\left(x_{i}, y_{j}\right)$ and $\Omega_{i, j}^{\beta}=\chi_{x}^{\beta}(i)+\chi_{y}^{\beta}(j)$ or $u_{i, j, k}=u\left(x_{i}, y_{j}, z_{k}\right), v_{i, j, k}=v\left(x_{i}, y_{j}, z_{k}\right)$ with $\Omega_{i, j, k}^{\beta}=\chi_{x}^{\beta}(i)+\chi_{y}^{\beta}(j)+\chi_{z}^{\beta}(k)$ in three dimensions. Boundary conditions are now set at extremes of the domain size $[-L, L]$. Now, the system has been converted to ODEs, the stiffness issue has equally been removed. It should be noted that any explicit higher-order time stepping integrators can be used.

\subsection{Adaptive exponential time differencing}

Exponential time differencing (ETD) schemes have a long history in the field of computational electrodynamics [32]. They have since gained tremendous attentions in various field of applied science, engineering and technology. Cox and Matthews [4] first derived a set of ETD schemes whose formulations were based on the $s$-stage Runge -Kutta time stepping integrators, which was called ETDRK methods. In the present paper, we utilize the fourth-order Runge-kuta-like method of Krogstad [14] and accommodate the fractional power in (7). The improved fractional order ETDRK4 formulae are

$$
\begin{aligned}
u_{n+1}= & u_{n} e^{\mathbf{L} h}+h\left[4 \varphi_{2}(\mathbf{L} h)-3 \varphi_{1}(\mathbf{L} h)+\varphi_{0}(\mathbf{L} h)\right] \mathbf{N}\left(u_{n}, v_{n}, t_{n}\right) \\
& +2 h\left[\varphi_{1}(\mathbf{L} h)-2 \varphi_{2}(\mathbf{L} h)\right] \mathbf{N}\left(a_{n}, t_{n}+h / 2\right) \\
& +2 h\left[\varphi_{1}(\mathbf{L} h)-2 \varphi_{2}(\mathbf{L} h)\right] \mathbf{N}\left(b_{n}, t_{n}+h / 2\right) \\
& +h\left[\varphi_{2}(\mathbf{L} h)-2 \varphi_{1}(\mathbf{L} h)\right] \mathbf{N}\left(c_{n}, t_{n}+h\right)
\end{aligned}
$$

and

$$
\begin{aligned}
a_{n}= & u_{n} e^{\mathbf{L} h / 2}+(\mathbf{L} h / 2) \varphi_{0}(\mathbf{L} h / 2) \mathbf{N}\left(u_{n}, v_{n}, t_{n}\right) \\
b_{n}= & u_{n} e^{\mathbf{L} h / 2}+(\mathbf{L} h / 2)\left[\varphi_{0}(\mathbf{L} h / 2)-2 \varphi_{1}(\mathbf{L} h / 2)\right] \mathbf{N}\left(u_{n}, v_{n}, t_{n}\right) \\
& +h \varphi_{1}(\mathbf{L} h / 2) \mathbf{N}\left(a_{n}, t_{n}+h / 2\right), \\
c_{n}= & u_{n} e^{\mathbf{L} h}+h\left[\left(\varphi_{0}(\mathbf{L} h)-2 \varphi_{1}(\mathbf{L} h)\right] \mathbf{N}\left(u_{n}, v_{n}, t_{n}\right)+2 h \varphi_{1}(\mathbf{L} h) \mathbf{N}\left(c_{n}, t_{n}+h\right),\right.
\end{aligned}
$$

where $\mathbf{L}=\Delta^{\beta}=\left(\partial / \partial x^{2}+\partial / \partial y^{2}\right)^{\beta}$ and $\mathbf{N}=\mathbf{N}(f(u, v), g(u, v))$ remain the linear and nonlinear operators. The functions $\varphi_{i}$ are given as

$$
\varphi_{0}(z)=\frac{e^{z}-1}{z}, \quad \varphi_{1}(z)=\frac{e^{z}-1-z}{z^{2}}, \quad \varphi_{2}(z)=\frac{e^{z}-1-z-z^{2} / 2}{z^{3}}
$$

are precisely the terms that emerge in the Lie group methods [17]. Details of derivation, stability and further applications can be found in $[4,11,14,21,22,23]$ and references therein.

\section{Numerical experiments}

In this section, we want to consider the nontrivial example of nonlinear Rosenzweig-MacArthur fractional partial differential equations for pattern formation in one and two dimensions. Numerical simulations of the di-trophic food-chain models are provided to demonstrate and compare the asymptotic nature of the time-dependent fractional reaction-diffusion system. 


\subsection{One-dimensional experiment}

We first illustrate the asymptotic stability of equilibrium $E_{s}$ result which correspond to the existence of the two species through numerical simulations of model (7) in absence of diffusion, that is $D_{u}=D_{v}=0$. In Figure 1, effect of the delay on the solution of the system (7) is shown at different instants of delay $\tau=0.5,1,1.5$ for columns 1,2,3 respectively. The first and second rows indicate the time series solutions and their corresponding limit cycles at time $t=100$. Clearly, increase in the delay $\tau$ has given rise to damped oscillations.

In Figure 2, we illustrate also the stability of the equilibrium $E_{s}$ via numerical simulations of the fractional reactiondiffusion system (7) at instants value of delay $\tau$, with ecological parameters: $\left\{\kappa=0.8, \sigma=4, \gamma=0.4, \delta=0.6, D_{u}=\right.$ $\left.0.2, D_{v}=0.05\right\}$. These parameters and initial populations $u(0)=0.5, v(0)=0.5$ are found satisfying the conditions for asymptotic stability in Section 2. It is noticeable that as $\tau$ is increased from 0.2 to 1.5 , both species oscillate in phase. So, the numerical simulation results in both Figures 1 and 2 agree with the theoretical result in Section 3.
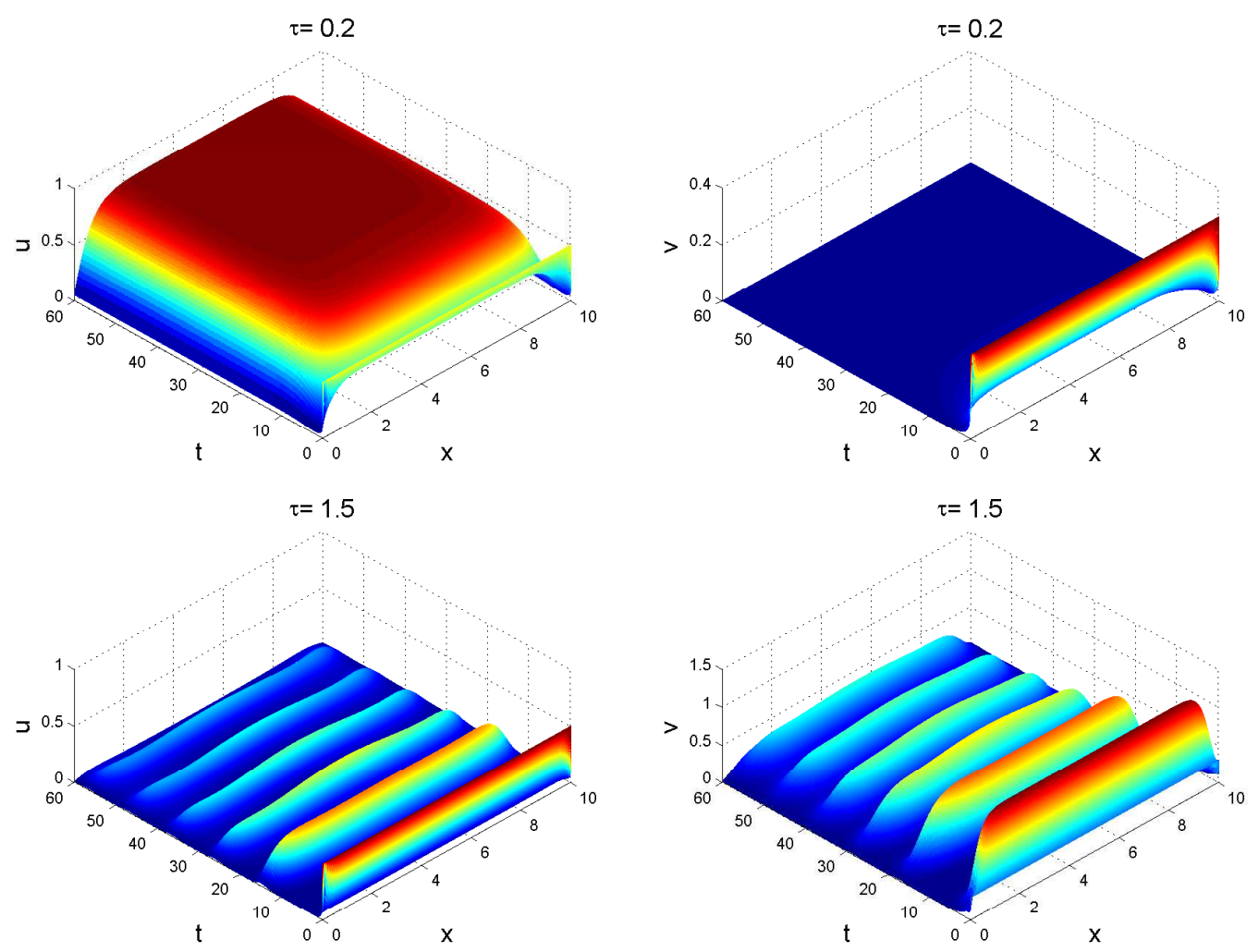

Figure 2. Surface plots showing the species evolution of Fractional reaction-diffusion system (7) at different instants of $\tau$. That is, $\tau=0.2,1.5$ with $\beta=1.2$ for upper- and lower-rows respectively.

Table 1. Convergence in space and computational time of the Fourier Spectral method at different instants of the fractional power $\beta$ at final time $t=1.0$ and $\tau=0.5$. The parameters are given in Figure 2

\begin{tabular}{|l|l|l|l|l|l|}
\hline & $N=32$ & $N=64$ & $N=128$ & $N=256$ & $N=512$ \\
\hline \multirow{2}{*}{$0.5=0.0761 \mathrm{e}-06$} & $2.2448 \mathrm{e}-08$ & $1.91433-08$ & $4.5501 \mathrm{e}-09$ & $2.1593 \mathrm{e}-09$ \\
& $0.0221 \mathrm{~s}$ & $0.0263 \mathrm{~s}$ & $0.0851 \mathrm{~s}$ & $0.0885 \mathrm{~s}$ & $0.1071 \mathrm{~s}$ \\
\hline \multirow{2}{*}{$\beta=1.0$} & $2.8384 \mathrm{e}-07$ & $6.0827 \mathrm{e}-09$ & $4.6338 \mathrm{e}-09$ & $8.2483 \mathrm{e}-10$ & $5.0311 \mathrm{e}-10$ \\
& $0.0015 \mathrm{~s}$ & $0.0043 \mathrm{~s}$ & $0.0891 \mathrm{~s}$ & $0.0 .1557 \mathrm{~s}$ & $0.4328 \mathrm{~s}$ \\
\hline \multirow{2}{*}{$\beta=1.5$} & $1.9144 \mathrm{e}-07$ & $9.0015 \mathrm{e}-09$ & $4.9107 \mathrm{e}-09$ & $8.8955 \mathrm{e}-10$ & $5.6208 \mathrm{e}-10$ \\
& $0.2001 \mathrm{~s}$ & $0.2032 \mathrm{~s}$ & $0.4305 \mathrm{~s}$ & $0.7871 \mathrm{~s}$ & $1.8203 \mathrm{~s}$ \\
\hline
\end{tabular}

To demonstrate the reliability and efficiency of our proposed method, we report the convergence results in space as well as the computational time for the fractional Rosenzweig-MacArthur system (7) for varying values of the fractional power $\beta$. Table 1 shows the convergence results of the fractional Fourier transform with different instants values of $\alpha$ at final time $t=1$. Reference solutions were calculated by evaluating (7) with $2^{10}$ Fourier modes. Obviously, the fractional Fourier approach is able to gain spectral convergence up to machine precision regardless of chosen $\beta$ values. 


\subsection{Two-dimensional experiment}

It is in higher dimensions that the methodology for fractional reaction-diffusion ideas presented in this paper really become of serious value. We illustrate the numerical algorithms using the nontrivial example. We simulate system (7), using initial conditions computed as

$\mathrm{u} 0=1-\exp \left(-10 *\left((\mathrm{xx}-1 / 2) \cdot{ }^{-2}+(\mathrm{yy}-1 / 2) \cdot{ }^{\wedge} 2\right)\right) ;$

$\mathrm{v} 0=\exp \left(-10 *\left((\mathrm{xx}-1 / 2) \cdot{ }^{\wedge} 2+2 *(\mathrm{yy}-1 / 2) \cdot{ }^{\wedge} 2\right)\right)$;

subject to boundary conditions clamped at both ends of the domain size $[-L, L]$.

In the simulation experiments, various type of dynamics are observed and we realiazed that both species have similar distributions. As a result, we streamlined our analysis of pattern formation to only one distribution (we report that of species $v$ in this paper). In Figure 3, we perform numerical simulations in $2 \mathrm{D}$ for different instants of fractional power $\beta=1,1.2,1.4$ in columns 1-3. We keep $L=5, D_{u}=2, D_{v}=0.05, \kappa=0.82, \sigma=4, \gamma=0.4, \delta=0.6$ and vary $\tau=0.21,0.52,1.05$ for rows 1-3 respectively. With the formation of spots pattern in the first row, it should be noted that formation of Turing and other patterns are possible depending on the choice of parameters and initial data. For instance, with parameter set $L=5$, $D_{u}=2, D_{v}=0.05, \kappa=0.86, \sigma=4.5, \gamma=0.34, \delta=0.65$, we obtain pure spots pattern in Figure 4 at $\beta=2$ which we clasify as sub-diffusive for $0<\beta<1$, diffusive for $\beta=1$ and super-diffusive in the region $1<\beta<2$.
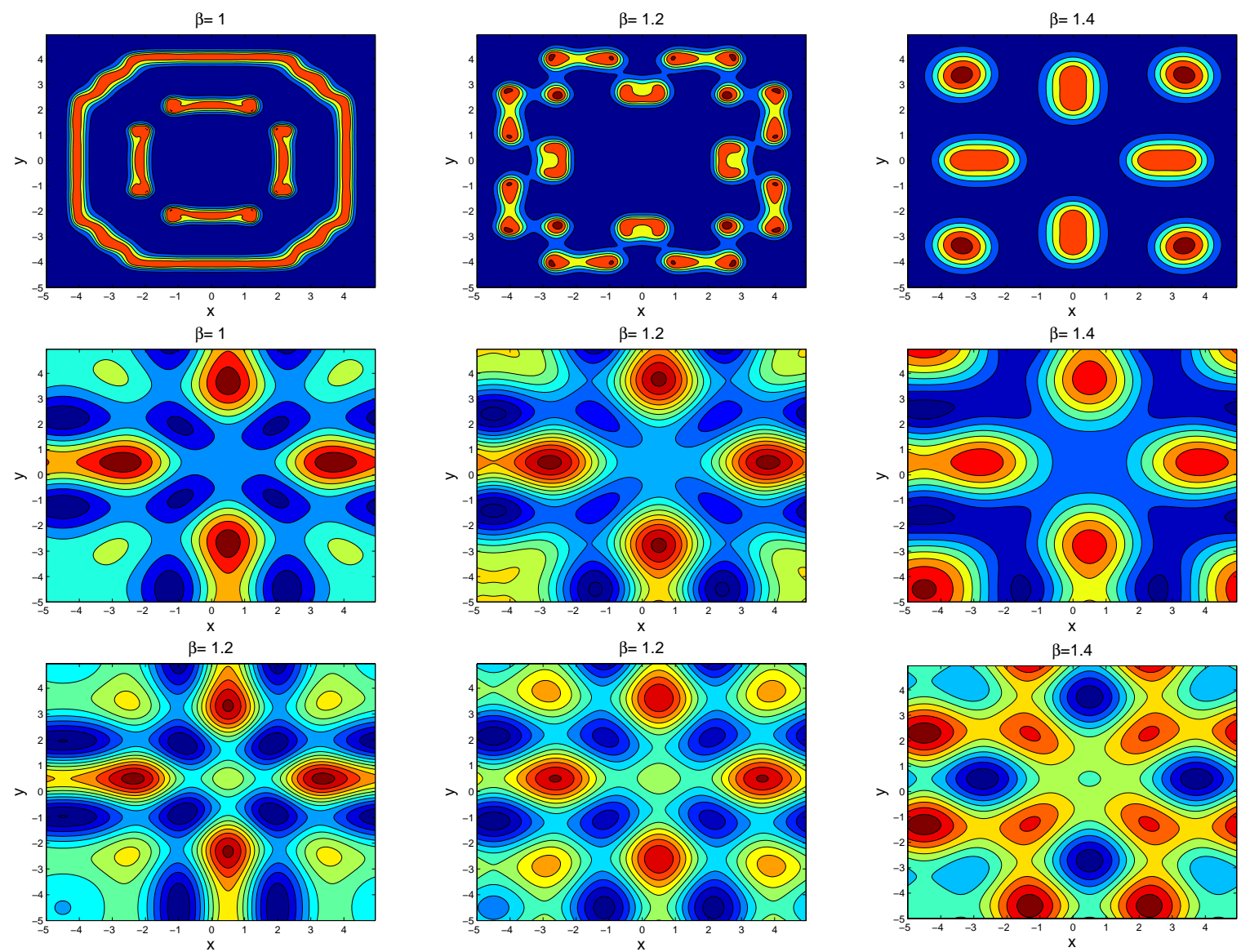

Figure 3. Contour plots of (7) in $2 \mathrm{D}$ showing effect of fractional power at different instants of $\beta=1,1.2,1.4$. The upper-, middle- and lower-rows correspond to $\tau=0.21,0.52,1.05$ respectively. Other parameters are: $D_{u}=2, D_{v}=0.05, \kappa=0.82, \sigma=4, \gamma=0.4$ and $\delta=0.6$ for simulation time $t=1000$. 

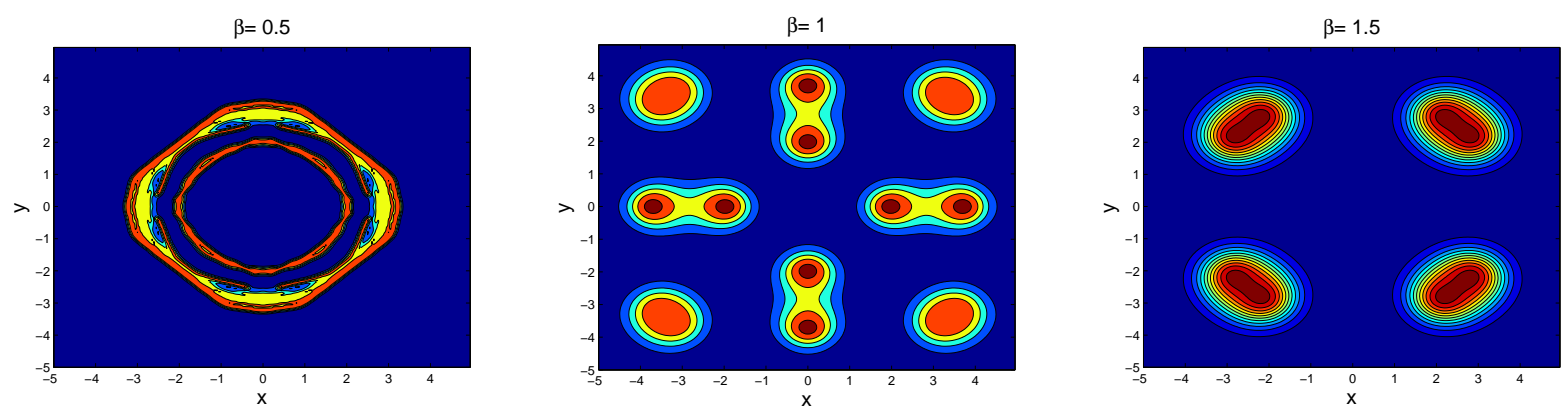

Figure 4. Contour plots of (7) in 2D showing effect of fractional power at different instants of $\beta=0.5,1,1.5$ which correspond to sub-diffusive, diffusive and super-diffusive scenarios at $t=1000$.

In Figure 5, we extend our numerical experiment of fractional Rosenzweig-MacArthur model to 3D. The initial conditions are computed as

$\mathrm{u} 0=1-\exp \left(-10 *\left((\mathrm{xx}-1 / 2) \cdot{ }^{\wedge} 2+(\mathrm{yy}-1 / 2) \cdot{ }^{\wedge} 2+(\mathrm{zz}-1 / 2) \cdot{ }^{\wedge} 2\right)\right)$;

$\mathrm{v} 0=\exp \left(-10 *\left((x x-1 / 2) \cdot{ }^{\wedge} 2+2 *(y y-1 / 2) \cdot{ }^{\wedge} 2+(z z-1 / 2) \cdot{ }^{\wedge} 2\right)\right)$;

in the clamped boundary of size $\pm L^{3}, L=10$. We observed that the distribution of both species are of the same type. The set of ecological parameters are chosen as:

$$
\left\{D_{u}=2, D_{v}=0.05, \tau=0.01, \kappa=5.0, \sigma=4.0, \gamma=0.4, \delta=0.6\right\} .
$$
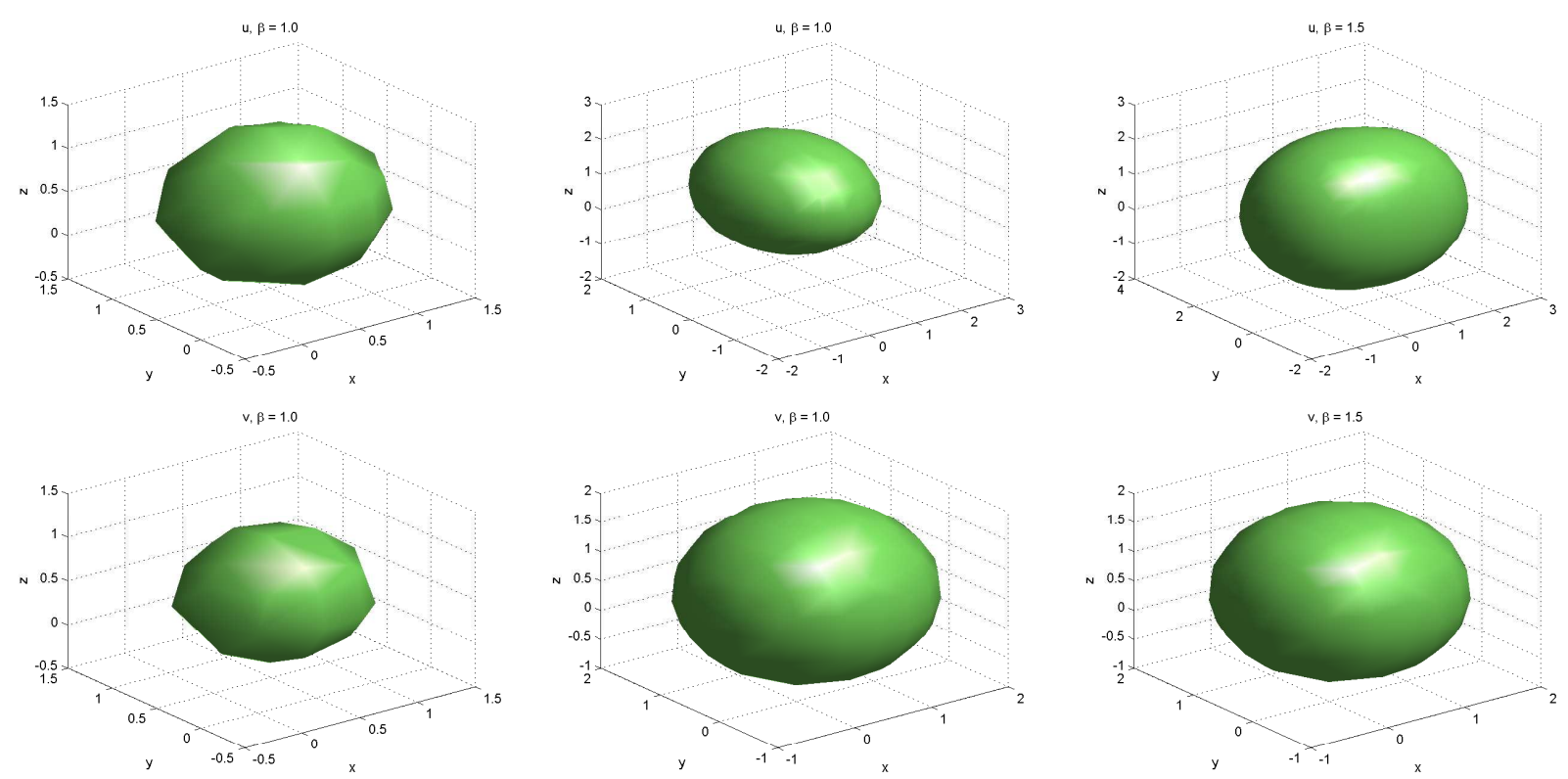

Figure 5. Numerical results of an extended space fractional Rosenzweig-MacArthur model (7) in 3D, showing the evolutions of both species $u$ (upper-row) and $v$ (lower-row) at different instants of the fractional power $\beta$ at $0.5,1.0$ and 1.5 which correspond to the sub-diffusive, diffusive and super-diffusive scenarios, at final time $t=5$. Other parameters are fixed in (12). 

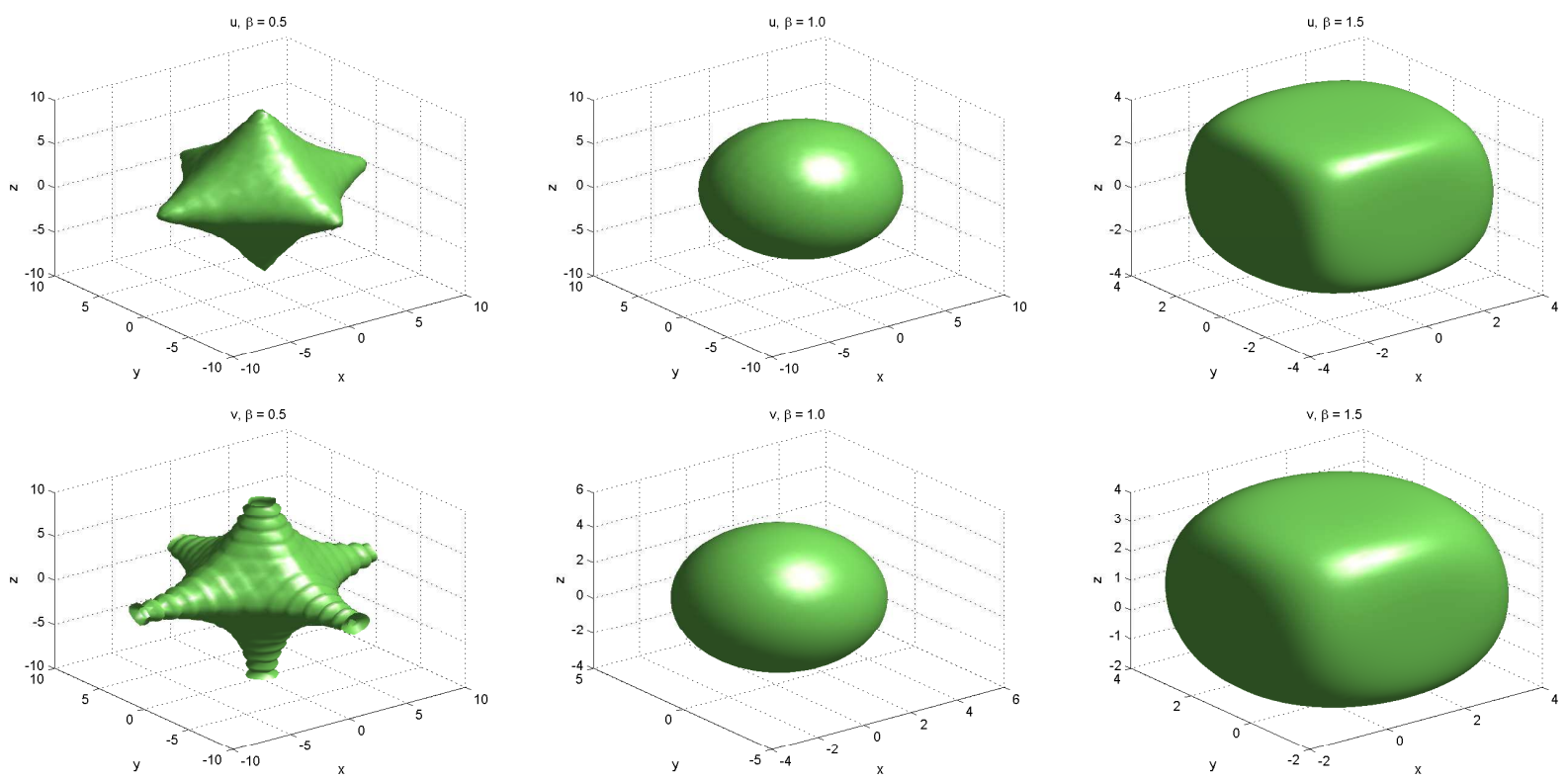

Figure 6. The 3D computer simulation results of an extended space fractional Rosenzweig-MacArthur model (7), showing the distribution of both species $u$ (upper-row) and $v$ (lower-row) at different instants of the fractional power $\beta$ at $0.5,1.0$ and 1.5 which correspond to the sub-diffusive, diffusive and super-diffusive scenarios, when the final time $t$ is increased from 5 to 50 . Other parameters are fixed in (12).

In Figure 6, we increase the simulation time from $t=5$ to $t=50$ to obtain various object patterns such as diamond, star, oval or egg-like and dice shapes. It should be mentioned that, with increasing time and perturbation of the initial data, other Turing dynamics can be observed.

\section{Conclusion}

In this work, we have demonstrate the use of Fourier spectral method in conjunction with the exponential time differencing scheme to numerically simulate the Rosenzweig-MacArthur fractional reaction-diffusion system with delay in the presence of fractional derivative index $\beta$. Our mathematical analysis results tallies with the numerical results as shown in Figures 1 and 2 , which justify the case for when the delay $\tau$ is increasing, both species oscillate in phase which can result to homogeneous and non-homogeneous oscillations. The 2D computer simulations of the fractional reaction-diffusion system (in the regions $\beta>1$ ) has given a strong proof that pattern formations in fractional case is practically the same as in the standard reactiondiffusion scenario at $\beta=1$. With the variation in the choice of parameters, one can observe the rich variety of spatiotemporal behaviour. The mathematical ideas presented in this work can be extended to solve both the integer and non-integer order fractional reaction-advection problems in one and high dimensions.

\section{Conflict of Interests}

The authors declare that there is no conflict of interests regarding the publication of this paper.

\section{REFERENCES}

[1] A. Atangana, On the stability and convergence of the time-fractional variable order telegraph equation, Journal of Computational Physics, 293 (2015) 104-114.

[2] Y. Chang, W. Feng, M. Freeze and X. Lu, Permanence and coexistence in a diffusive complex ratio-dependent food chain, International Journal of Dynamical and Control Systems DOI:10.1007/s40435-014-0131-4 (2014).

[3] W. Chen, L. Ye and H. Sun, Fractional diffusion equations by Kansa method, Computers and Mathematics with Applications 59 (2010) 1614-1620.

[4] S.M. Cox and P.C. Matthews, Exponential time differencing for stiff systems, Journal of Computational Physics 176 (2002) $430-455$. 
[5] L. Edelstein-Keshet, Mathematical Models in Biology, SIAM, New York, 1988.

[6] W. Feng, N. Rocco, M. Freeze and X. Lu, Mathematical analysis on an extended Rosenzweig-MacArthur model of tri-trophic food chain, Discrete and Continuous Dynamical Systems- Series $S 7$ (2014) 1215-1230.

[7] B. Fornberg, A practical guide to Pseudospectral methods, Cambridge University Press, Cambridge, 1998.

[8] B. Fornberg and Driscoll, A fast spectral algorithm for nonlinear wave equations with linear dispersion, Journal of Computational Physics 155 (1999) 456-467.

[9] M. Garg and P. Manohar, Numerical solution of fractional diffusion-wave equation with two space variables by matrix method, Fractional Calculus and Applied Analysis, 13 (2010) 191-207.

[10] L. Hu, D. Chen and G.W. Wei, High-order fractional partial differential equation transform for molecular surface construction, Molecular Based Mathematical Biology 1 (2013) 1-25.

[11] A.K. Kassam and L.N. Trefethen, Fourth-order time-stepping for stiff PDEs. SIAM Journal of Scientific Computing 26 (2005) 12141233.

[12] A.A. Kilbas, Yu.F. Luchko, H. Martnezc and J.J. Trujillod, Fractional Fourier transform in the framework of fractional calculus operators Integral Transforms and Special Functions, 21 (2010) 779-795.

[13] A.A. Kilbas, H.M. Srivastava and J.J. Trujillo, Theory and Applications of Fractional Differential Equations, Elsevier, Netherlands, 2006.

[14] S. Krogstad, Generalized integrating factor methods for stiff PDEs, Journal of Computational Physics 203 (2005) $72-88$.

[15] Q. Liu, F. Liu, Y. Gu, P. Zhuang, J. Chen and I. Turner, A meshless method based on Point Interpolation Method (PIM) for the space fractional diffusion equation, Applied Mathematics and Computation, 256 (2015) 930-938.

[16] R. May, Complexity and stability in model ecosystems, Princeton University Press, 1973.

[17] H. Munthe-Kaas, High order Runge-Kutta methods on manifolds, Applied Numerical Mathematics 29 (1999) $115-127$.

[18] K.M. Owolabi, Robust IMEX schemes for solving two-dimensional reaction-diffusion models, International Journal of Nonlinear Science and Numerical Simulations 16 (2015) 271-284. DOI 10.1515/ijnsns-2015-0004.

[19] K.M. Owolabi and K.C. Patidar, Numerical simulations of multicomponent ecological models with adaptive methods, Theoretical Biology and Medical Modelling 13 (2016), 1. DOI 10.1186/s12976-016-0027-4.

[20] K.M. Owolabi and K.C. Patidar, Existence and permanence in a diffusive KiSS modelwith robust numerical simulations, International Journal of Differential Equations (2015); 2015(485860):8. doi:10.1155/2015/485860.

[21] K.M. Owolabi and K.C. Patidar, Numerical solution of singular patterns in one-dimensional Gray-Scott-like models, International Journal of Nonlinear Science and Numerical Simulations 15 (2014) 437-462. DOI 10.1515/ijnsns-2013-0124.

[22] K.M. Owolabi and K.C. Patidar, Higher-order time-stepping methods for time-dependent reaction-diffusion equations arising in biology, Applied Mathematics and Computation 240 (2014), 30-50.

[23] K.M. Owolabi and K.C. Patidar, Effect of spatial configuration of an extended nonlinear KiersteadSlobodkin reactiontransport model with adaptive numerical scheme, Springer Plus (2016) 5:303. DOI 10.1186/s40064-016-1941-y

[24] H.K. Pang and H.W. Sun, Multigrid method for fractional diffusion, Journal of Computational Physics, 231 (2012) $693-703$.

[25] E. Pindza, K.M. Owolabi, Fourier spectral method for higher order space fractional reaction-diffusion equations, Communications in Nonlinear Science and Numerical Simulation (2016), doi: 10.1016/j.cnsns.2016.04.020

[26] I. Podlubny, A. Chechkin, T. Skovranek, Y.Q. Chen and B.B. Jara, Matrix approach to discrete fractional calculus II: Partial fractional differential equations, Journal of Computational Physics, 228 (2009) 3137-3153.

[27] I. Podlubny, Fractional differential equations, Academic Press, New York, 1999.

[28] A.D. Polyanin and V.F. Zaitsev, Handbook of Nonlinear Partial Differential Equations, Chapman \& Hall/CRC, Boca Raton, FL, 2004.

[29] M. L. Rosenzweig and R. H. MacArthur, Graphical representation and stability conditions of predator-prey interactions, American Naturalist 97 (1963) 209-223.

[30] G. Sun, G. Zhang, Z. Jin, Q. Liu and L. Li, Dynamical complexity of a spatial predator-prey model with migration, Ecological Modelling 219 (2008) 248-255. 
[31] G. Sun, G. Zhang, Z. Jin and L. Li, Predator cannibalisms can give rise to regular spatial patterns in a predator-prey system, Nonlinear Dynamics 58 (2009) 75-84.

[32] A. Taflove, Computational electrodynamics: the finite difference time-domain method, Artech House, Boston, 1995.

[33] L.N. Trefethen, Spectral Methods in MATLAB. SIAM Publications, Philadelphia, 2000.

[34] V. Volpert and S. Petrovskii, Reaction-diffusion waves in biology, Physics of Life Reviews 6 (2009) 267-310.

[35] H. Wang, K. Wang and T. Sircar, A direct O $\left(N \log _{2} N\right)$ finite difference method for fractional diffusion equations, Journal of Computational Physics, 229 (2010) 8095-8104.

[36] H. Wang and N. Du, A super fast-preconditioned iterative method for steady-state space fractional diffusion equations, Journal of Computational Physics 240 (2013) 49-57.

[37] J.A.C. Weideman and S.C. Reddy, A MATLAB differenciation suite, Transactions on Mathematical Software 26 (2001), $465-519$.

[38] D.M. Wrzosek, Limit cycles in predator models, Mathematical Biosciences 98 (1990) 1-12.

[39] F. Zeng, C. Li, F. Liu, and I. Turner, Numerical algorithms for time-fractional subdiffusion equation with second-order accuracy, SIAM Journal on Scientific Computing, 37 (2015) A55-A78.

[40] F. Zeng, F. Liu, C. Li, K. Burrage, I. Turner and V. Anh, A Crank-Nicolson ADI spectral method for a two-dimensional riesz space fractional nonlinear reaction-diffusion equation, SIAM Journal on Numerical Analysis, 52 (2014) 2599-2622.

[41] M. Zheng, F. Liu, I. Turner and V. Anh, A Novel High Order Space-Time Spectral Method for the Time Fractional Fokker-Planck Equation, SIAM Journal on Scientific Computing, 37 (2015), A701-A724.

[42] Y. Zhou, Basic Theory of Fractional Differential Equations, World Scientific, New Jersey, 2014. 